2005, 109, 11427-11431

Published on Web 05/18/2005

\title{
Absolute Standard Redox Potential of Monolayer-Protected Gold Nanoclusters
}

\author{
Bin Su and Hubert H. Girault* \\ Laboratoire d'Electrochimie Physique et Analytique, Ecole Polytechnique Fédérale de Lausanne, Station 6, \\ CH-1015 Lausanne, Switzerland
}

Received: March 21, 2005; In Final Form: April 28, 2005

\begin{abstract}
The redox properties of monolayer-protected gold nanoclusters (MPCs) are considered from both the theoretical and experimental viewpoints. The "absolute standard redox potential" $\left(\left[E_{Z / Z-1}^{0}\right]_{\text {abs }}\right)$ of MPCs is first derived from electrostatic considerations. A linear dependence of the absolute standard redox potential on the valence state of MPCs is theoretically predicted and verified experimentally. By employing ferricinium/ferrocene $\left(\mathrm{Fc}^{+} / \mathrm{Fc}\right)$ as a reference redox couple, the average valence state of MPCs at a given potential can be estimated.
\end{abstract}

\section{Introduction}

Alkanethiolate stabilized gold nanoparticles prepared using the Brust reaction, ${ }^{1,2}$ so-called monolayer-protected gold nanoclusters (MPCs), have received considerable interest owing to their unique electronic and chemical properties, notably the successive single-electron transfer characteristics. ${ }^{3-6}$ At ambient temperature, both freely diffusing and electrode-attached MPCs demonstrate the voltammetric responses featuring a series of evenly spaced current peaks.,7-21 This ensemble behavior is equivalent to a series of classical Coulomb staircase charging events or sequential electrochemical redox reactions, which occur as MPCs encompass an intermediate dimension between small molecules and bulk materials. Previously, the sequential electron transfer has been observed for fullerene (and derivatives) $)^{22-26}$ and Pt-carbonyl nanoclusters $\left[\mathrm{Pt}_{n}(\mathrm{CO})_{m}\right],{ }^{27-29}$ which led Weaver et al. ${ }^{30}$ to develop an electrostatic model to relate the electron transfer energetics of molecular capacitances in gas- and solution-phase systems. On the basis of this model, Chen et al. ${ }^{8}$ ascribed the occurrence of the successive electron transfer for MPCs to the extremely small (sub-attoFarad, aF) molecular capacitances of MPCs associated with a combination of a small metallic core and a dielectric protecting layer. The capacitance of an MPC with a core size smaller than $2 \mathrm{~nm}$ is less than 1 attofarad $(\mathrm{aF})$, which is so small that a single electron addition to or removal from its core produces a substantial potential change. Accordingly, the sequential electron transfer event involving MPCs has been termed quantized double-layer charging. Nevertheless, this approach assumes that the MPC can be treated as a metallic phase with an inner or Galvani potential. An alternative approach is to consider an MPC as a "giant molecule" and to approximate electron transfers as classical reactions.

Extensive electrochemical studies have shown that MPCs are formally equivalent to multivalent redox species which exhibit equally spaced formal redox potentials: the charged metallic cores can be used as electron donors and acceptors in electron transfer reactions. Furthermore, MPCs can be electrochemically charged to the desired valence state in solutions. The charged MPCs are stable and can even be isolated in a solid form. ${ }^{7}$ This

* Corresponding author: Prof. Hubert H. Girault. Phone: +41-21 693 3151. Fax: +41-21 693 3667. E-mail: hubert.girault@epfl.ch. property enables MPCs to act as tunable and quantitative redox probes in electron transfer chemistry. ${ }^{6}$ Given their unique electrochemical property and reactivity, it is of fundamental interest to understand the factors determining the sequential electron transfer energetics and to relate them to the energy scale of a certain reference system. In this work, the relation between the absolute standard redox potential $\left(\left[E_{Z / Z-1}^{0}\right]_{\mathrm{abs}}\right)$ of a charged state "couple" and its valence state is developed and verified by voltammetry on a solid electrode. By employing ferricinium/ ferrocene $\left(\mathrm{Fc}^{+} / \mathrm{Fc}\right)$ as a reference redox couple, the average charge state of MPCs at a given potential can be estimated.

\section{Experimental Section}

All chemicals employed were of the highest commercially available purity and used as received. Hydrogen tetrachloroaurate trihydrate $\left(\mathrm{HAuCl}_{4} \cdot 3 \mathrm{H}_{2} \mathrm{O}\right)$, tetraoctylammonium bromide (TOABr), sodium tetrahydroborate $\left(\mathrm{NaBH}_{4}\right)$, and 1-hexanethiol $(\mathrm{C} 6-\mathrm{SH})$ were obtained from Aldrich. Ethanol, acetonitrile $\left(\mathrm{CH}_{3} \mathrm{CN}\right)$, dichloromethane, 1,2-dichloroethane (DCE), bis(triphenylphosphoranylidene)ammonium chloride (BTPPACl), and ferrocene $(\mathrm{Fc})$ were provided by Fluka. Lithium tetrakis(pentafluorophenyl)borate diethyl etherate (LiTPFB) was purchased from Boulder Scientific Company. The organic supporting electrolyte, bis(triphenylphosphoranylidene) ammonium tetrakis(pentafluorophenyl)borate (BTPPATPFB), was prepared as previously reported. ${ }^{31}$

The MPCs were prepared by the Brust reaction ${ }^{1,2}$ followed by extraction and annealing processes to harvest a population of MPCs with relatively uniform metallic cores. ${ }^{16}$ Briefly, 1.55 $\mathrm{g}$ of $\mathrm{HAuCl}_{4} \cdot 3 \mathrm{H}_{2} \mathrm{O}$ in water was mixed with $1.12 \mathrm{~g}$ of $\mathrm{TOABr}$ in toluene under vigorous stirring. After $\mathrm{AuCl}_{4}{ }^{-}$was completely extracted into toluene, $1.8 \mathrm{~mL}$ C6-SH corresponding to a 3:1 molar ratio $(\mathrm{S} / \mathrm{Au})$ was added. The mixture was further stirred for $20 \mathrm{~min}$, followed by the addition of $1.90 \mathrm{~g}$ of $\mathrm{NaBH}_{4}$ all at once at $0{ }^{\circ} \mathrm{C}$, which resulted in an immediate, pronounced darkening of the mixture. The reduction was allowed to proceed for $45 \mathrm{~min}$, after which the water layer was removed with a separating funnel and the toluene removed by rotary evaporation at $40{ }^{\circ} \mathrm{C}$. The black slurry remaining in the round-bottom flask includes all fractions of MPCs, byproducts, and unreacted residues. The extraction was then carried out as follows: ethanol 

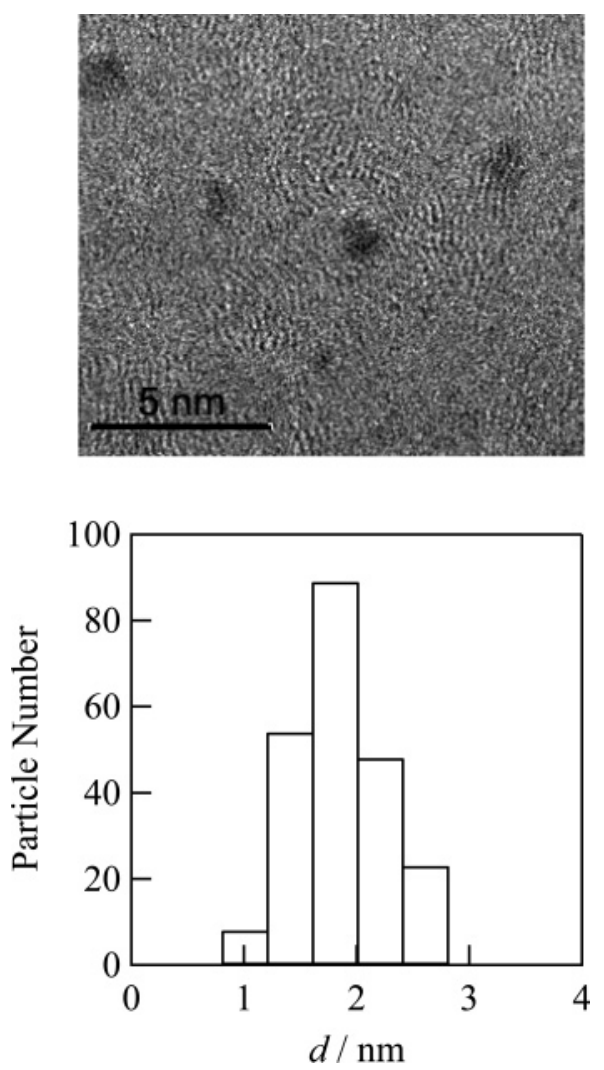

Figure 1. HRTEM image (top) and the corresponding size distribution (bottom) of a sample of MPCs.

was added to the flask, which was covered to stand overnight and then filtered. The filtrate was rotovaped to dryness and then redispersed in $\mathrm{CH}_{3} \mathrm{CN}$. After $24 \mathrm{~h}$, the MPC product was collected on a frit where it was washed with copious amounts of $\mathrm{CH}_{3} \mathrm{CN}$. The extraction treatment removed not only the byproducts and unreacted residues but also some fractions of smaller core size MPCs. ${ }^{14}$ The obtained MPCs were further annealed in dichloromethane by adding a 500-fold excess of C6-SH versus MPC dissolved. After stirring for 4 days, dichloromethane was evaporated. The remaining product was sonicated in $\mathrm{CH}_{3} \mathrm{CN}$ and collected through several cycles of centrifugation and washing. The final product has a dominant population with an average core mass of $28 \mathrm{kDa}$, which corresponds to ca. $140 \mathrm{Au}$ atoms protected with ca. 53 hexanethiolate ligands. ${ }^{16}$ The population has a mean diameter of $1.6 \pm 0.4 \mathrm{~nm}$ in the metallic core determined by highresolution transmission electron microscopy (HRTEM) (Philips, CM 300), as shown in Figure 1.

Differential pulse voltammetry (DPV) of MPCs in the DCE electrolyte solution (ca. $0.02 \mathrm{mM}$ MPC, $0.01 \mathrm{M}$ BTPPATPFB) was performed on a $\mathrm{CH}-900$ Electrochemical Workstation $(\mathrm{CH}-$ Instruments, TX). A two-electrode arrangement was used, in which a silver wire was used both as quasi-reference electrode (QRE) and counter electrode. The working electrode was a 25$\mu$ m-diameter disk-shaped Pt microelectrode $(\mathrm{CH}$-Instruments, TX), which was rinsed with water and acetone and dried prior to each measurement.

\section{Results and Discussion}

Figure 2 schematically illustrates an MPC embedded in a dielectric medium. An MPC is considered here as a concentric sphere consisting of a spherical nanometer-sized metallic core of radius $r_{0}$ and a coating ligand monolayer that behaves as a dielectric layer of thickness $d$ with a dielectric constant $\epsilon_{\mathrm{d}}$. The

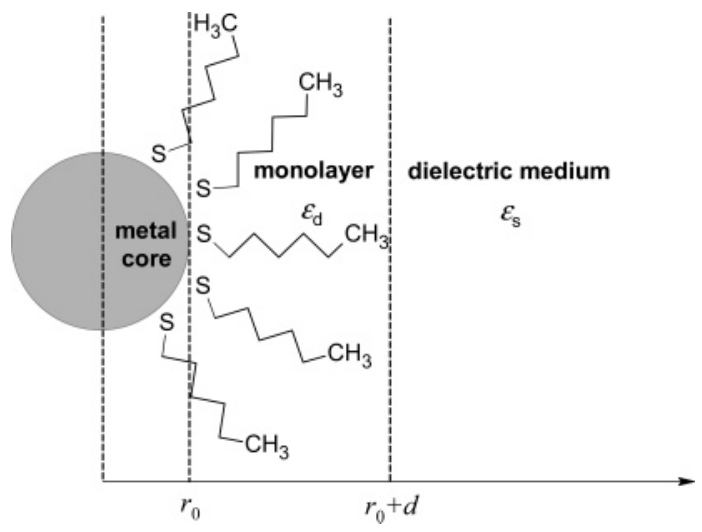

Figure 2. Schematic illustration of the concentric structure of an MPC embedded in a dielectric medium.

electric field inside the ligand monolayer and outside the sphere are defined, respectively, as

$$
\begin{aligned}
& E_{1}=-\frac{\mathrm{d} V}{\mathrm{~d} r}=\frac{q}{4 \pi \epsilon_{0} \epsilon_{\mathrm{d}} r^{2}} \quad\left(r_{0} \leq r \leq r_{0}+d\right) \\
& E_{2}=-\frac{\mathrm{d} V}{\mathrm{~d} r}=\frac{q}{4 \pi \epsilon_{0} \epsilon_{\mathrm{s}} r^{2}} \quad\left(r_{0}+d \leq r<\infty\right)
\end{aligned}
$$

where $r$ is the distance from the center of the metallic core and $q$ the charge that the MPC carries. With spherical coordinates and the potential equal to zero when $r \rightarrow \infty$, the electrostatic potential at the surface of the metallic core corresponds to the sum of the integrals of eqs 1 and 2

$$
\begin{aligned}
V(q) & =\int_{r_{0}}^{r_{0}+d} E_{1} \mathrm{~d} r+\int_{r_{0}+d}^{\infty} E_{2} \mathrm{~d} r \\
& =\frac{q}{4 \pi \epsilon_{0}}\left[\left(\frac{1}{\epsilon_{\mathrm{s}}}-\frac{1}{\epsilon_{\mathrm{d}}}\right)\left(\frac{1}{r_{0}+d}\right)+\frac{1}{\epsilon_{\mathrm{d}} r_{0}}\right]
\end{aligned}
$$

When $d$ is zero, eq 3 reduces to the potential at the surface of a bare sphere in a dielectric environment.

Considering MPCs as multivalent redox species, the sequential one-electron transfer process can be generally depicted as an electrochemical reaction at an electrode

$$
\mathrm{MPC}^{Z-1}-\mathrm{e}^{-} \rightleftharpoons \mathrm{MPC}^{Z}
$$

The absolute standard redox potential can be expressed either from the real chemical potentials of the oxidized and reduced species $^{32}$ or from a thermodynamic cycle. As shown in Figure $3 \mathrm{a}$, the one-electron oxidation reaction can be decomposed to three steps: the transfer of $\mathrm{MPC}^{\mathrm{Z}-1}$ from the solvent phase to the gas phase, the ionization of $\mathrm{MPC}^{Z-1}$ to form $\mathrm{MPC}^{Z}$ in the gas phase, and the transfer of $\mathrm{MPC}^{Z}$ from the gas phase to the solvent phase. At a first approximation, the work to transfer a charged sphere from the gas phase to a solvent phase can be considered equal to the Gibbs solvation energy of the charged sphere. Therefore, the absolute standard redox potential, [ $\left.E_{Z / Z-1}^{0}\right]_{\mathrm{abs}}$, is given by

$$
\begin{aligned}
e\left[E_{\mathrm{Z} / \mathrm{Z}-1}^{0}\right]_{\mathrm{abs}} & =\alpha_{\mathrm{MPCZ}}^{0}-\alpha_{\mathrm{MPC}-1}^{0} \\
& =\Delta G_{\mathrm{solv}, \mathrm{MPCZ}}^{0}-\Delta G_{\mathrm{solv}, \mathrm{MPCZ}-1}^{0}+E_{\mathrm{I}, Z-1}^{0}
\end{aligned}
$$

where $e$ is the elementary electronic charge and $E_{\mathrm{I}, Z-1}^{0}$ the ionization energy of $\mathrm{MPC}^{\mathrm{Z}-1}$ in the gas phase. $\alpha_{\mathrm{MPCZ}}^{0, Z-1}$ and 

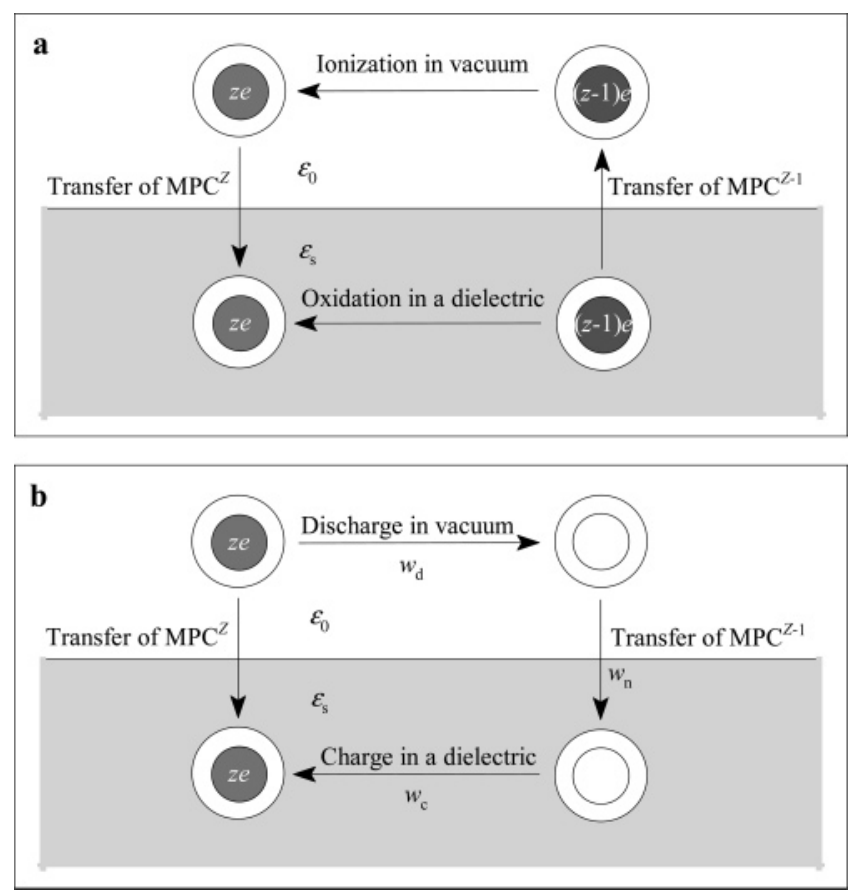

Figure 3. (a) Equivalence of a single-electron oxidation process in a solvent phase to a cycle of an ionization process in the vacuum preceded and followed by transfer of a charged-state couple of MPC between the vacuum and the solvent phase; (b) Born's model of the solvation of $\mathrm{MPC}^{Z}$

$\alpha_{\mathrm{MPCZ}-1}^{0}$ are the real chemical potentials of $\mathrm{MPC}^{\mathrm{Z}}$ and $\mathrm{MPC}^{\mathrm{Z}-1}$, respectively. $\Delta G_{\text {solv,MPCZ }}^{0}$ and $\Delta G_{\text {solv,MPCZ-1 }}^{0}$ represent the standard Gibbs solvation energies of $\mathrm{MPC}^{\mathrm{Z}}$ and $\mathrm{MPC}^{\mathrm{Z}-1}$, respectively.

Evaluation of $\Delta G_{\mathrm{solv}, \mathrm{MPCZ}}^{0}$ can be done on the basis of Born's model of ionic solvation, as illustrated in Figure $3 \mathrm{~b}$. The difference between an MPC ${ }^{Z}$ and a conventional ion is that the $\mathrm{MPC}^{Z}$ contains an intrinsic dielectric coating layer. From Figure $3 \mathrm{~b}$, the work of transferring $\mathrm{MPC}^{Z}$ from the gas phase to the solvent phase $\left(\Delta G_{\mathrm{solv}, \mathrm{MPC}}^{0}\right)$ corresponds to the sum of the work of discharging $\mathrm{MPC}^{\mathrm{Z}}$ in a vacuum to form a neutral sphere of the same size $\left(w_{\mathrm{d}}^{0}\right)$, the work of transferring this neutral sphere from the vacuum to the phase $\left(w_{\mathrm{n}}\right)$, and the work of charging this sphere in the solvent phase $\left(w_{\mathrm{c}}^{\mathrm{s}}\right)$ :

$$
\Delta G_{\mathrm{solv}, \mathrm{MPCZ}}^{0}=w_{\mathrm{d}}^{0}+w_{\mathrm{c}}^{\mathrm{s}}+w_{\mathrm{n}}
$$

with

$$
\begin{array}{r}
w_{\mathrm{c}}^{\mathrm{s}}=\int_{0}^{z e} V(q) \mathrm{d} q=\frac{z^{2} e^{2}}{8 \pi \epsilon_{0}}\left[\left(\frac{1}{\epsilon_{\mathrm{s}}}-\frac{1}{\epsilon_{\mathrm{d}}}\right)\left(\frac{1}{r_{0}+d}\right)+\frac{1}{\epsilon_{\mathrm{d}} r_{0}}\right] \\
w_{\mathrm{d}}^{0}=-\int_{0}^{z e} V(q) \mathrm{d} q=-\frac{z^{2} e^{2}}{8 \pi \epsilon_{0}}\left[\left(1-\frac{1}{\epsilon_{\mathrm{d}}}\right)\left(\frac{1}{r_{0}+d}\right)+\frac{1}{\epsilon_{\mathrm{d}} r_{0}}\right]
\end{array}
$$

Substituting eqs 7 and 8 into eq 6 , we obtain

$$
\Delta G_{\mathrm{solv}, \mathrm{MPCZ}}^{0}=\frac{z^{2} e^{2}}{8 \pi \epsilon_{0}}\left(\frac{1}{\epsilon_{\mathrm{s}}}-1\right)\left(\frac{1}{r_{0}+d}\right)+w_{\mathrm{n}}
$$

The ionization energy of $\mathrm{MPC}^{Z-1}$ in a vacuum $\left(E_{\mathrm{I}, Z-1}^{0}\right)$ can be expressed as ${ }^{30,33}$

$$
E_{\mathrm{I}, Z-1}^{0}=\Phi+w_{\mathrm{c}}^{0, Z-1 \rightarrow Z}
$$

where $\Phi$ is the work function of the metal, which is equal to $5.32 \mathrm{eV}$ for bare gold. ${ }^{32}$ In the presence of adsorption of alkanethiol monolayers on the surface, $\Phi$ will be changed because of the formation of a gold-sulfur dipole layer. In the case of 1-hexanethiol, the work function is varied by less than $100 \mathrm{mV}$ for the planar Au electrode. ${ }^{34}$ For the present MPCs, we can therefore neglect the contribution of the monolayer to the surface potential. The second term in eq $10, w_{\mathrm{c}}^{0, Z-1 \rightarrow Z}$, is equal to the work of charging an MPC from charged state $z-$ 1 to $z$ in a vacuum

$$
\begin{array}{r}
w_{\mathrm{c}}^{0, Z-1 \rightarrow Z}=\int_{(z-1) e}^{z e} V(q) \mathrm{d} q=\frac{(2 z-1) e^{2}}{8 \pi \epsilon_{0}}\left[\frac{1}{\epsilon_{\mathrm{d}}}\left(\frac{1}{r_{0}}-\frac{1}{r_{0}+d}\right)+\right. \\
\left.\left(\frac{1}{r_{0}+d}\right)\right]
\end{array}
$$

From eqs 10 and 11, we get

$$
E_{\mathrm{I}, Z-1}^{0}=\Phi+\frac{(2 z-1) e^{2}}{8 \pi \epsilon_{0}}\left[\frac{1}{\epsilon_{\mathrm{d}}}\left(\frac{1}{r_{0}}-\frac{1}{r_{0}+d}\right)+\left(\frac{1}{r_{0}+d}\right)\right]
$$

For a certain MPC radius, eq 12 predicts that the ionization energy linearly increases with $z$, which reflects the increasing energy cost of electron loss arising from the electrostatic interactions. Here, we must note that an MPC differs from a conventional multivalent redox molecule, because the latter possesses discrete electronic energy levels, which correspond to discrete ionization potentials that are not generally equally spaced. MPCs containing $<200 \mathrm{Au}$ atoms are in an intermediate regime between molecular and bulk materials, where electronic band energetics become size- and surface-confined, and a series of electronic states emerge and distribute in relatively uniform spacing in a certain energetic range.

From eqs 5, 9, and 12, the absolute standard redox potential, $\left[E_{Z / Z-1}^{0}\right]_{\mathrm{abs}}$, can then be derived as

$$
\left[E_{\mathrm{Z} / \mathrm{Z}-1}^{0}\right]_{\mathrm{abs}}=\frac{\Phi_{\mathrm{b}}}{e}+\frac{\left(z-\frac{1}{2}\right) e}{4 \pi \epsilon_{0}\left(r_{0}+d\right)}\left(\frac{d}{\epsilon_{\mathrm{d}} r_{0}}+\frac{1}{\epsilon_{\mathrm{s}}}\right)
$$

Equation 13 differs with that proposed by Chen et al. ${ }^{8}$ on the basis of the electrostatic model described earlier by Weaver et al. ${ }^{30}$

$$
E_{\mathrm{Z} / \mathrm{Z}-1}^{0}=E_{\mathrm{PZC}}+\frac{\left(z-\frac{1}{2}\right) e}{4 \pi \epsilon_{0} \epsilon_{\mathrm{d}}\left(\frac{r_{0}}{d}\right)\left(r_{0}+d\right)}
$$

where $E_{\mathrm{PZC}}$ is defined as the potential of zero charge $(z=0)$ of the MPC. However, eq 14 gives rise to two concerns. First, the standard redox potential relies on the determination of $E_{\mathrm{PZC}}$ that is medium-dependent and difficult to measure. This determination has been carried out by impedance measurements of a monolayer attached to an Au electrode. ${ }^{9}$ Indeed, the capacitance data measured refer to a planar electrode covered by a layer of 4,4'-thiobisbenzenethiol. Even if the capacitance minimum observed is the potential of zero charge, that is, if we assume the absence of any ionic-specific adsorption on the planar gold electrode, it does not necessary mean that the MPCs are neutral. It just means that the diffuse layer of MPCs and other ions is 

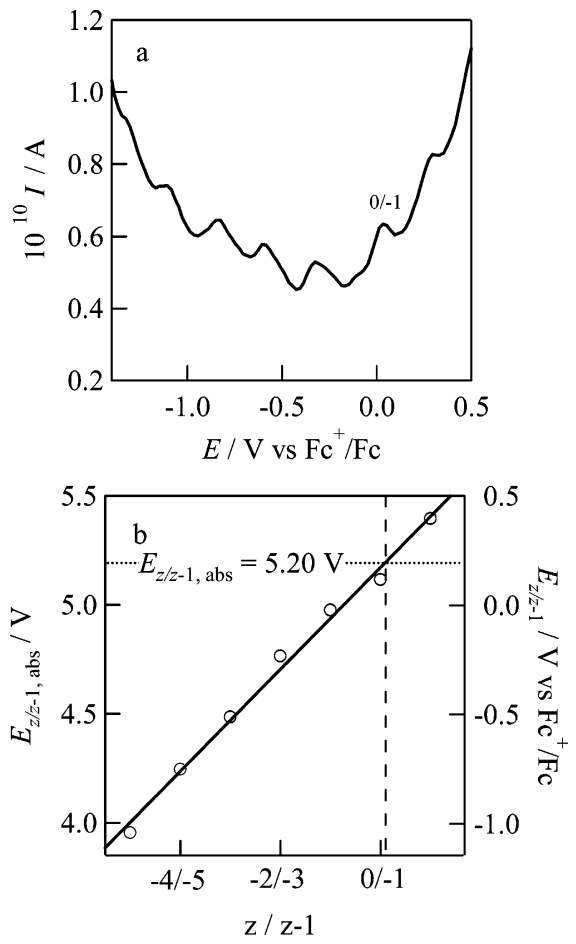

Figure 4. (a) A typical DPV response of MPCs in the 1,2dichloroethane electrolyte solution (ca. $0.02 \mathrm{mM}$ MPCs and $0.01 \mathrm{M}$ BTPPATPFB) measured at a $25-\mu \mathrm{m}$ Pt electrode: scan rate $20 \mathrm{mV}$ $\mathrm{s}^{-1}$, pulse height $50 \mathrm{mV}$, pulse width $60 \mathrm{~ms}$, and period $200 \mathrm{~ms}$. (b) The corresponding $Z$-plot of $\left[E_{Z / Z^{-1}}^{0}\right]_{\text {abs }}$ vs the adjacent valence states.

globally neutral. The $E_{\mathrm{PZC}}$ of a bulk metal in the absolute scale is linked to the work function of the metal by ${ }^{32}$

$$
E_{\mathrm{PZC}}=\frac{\Phi}{e}+\Delta_{\mathrm{S}}^{\mathrm{M}} g(\operatorname{dip})+\chi^{\mathrm{S}}-\chi^{\mathrm{M}}
$$

where $\Delta_{\mathrm{S}}^{\mathrm{M}} g(\operatorname{dip}), \chi^{\mathrm{S}}$, and $\chi^{\mathrm{M}}$ represent the dipolar contribution to the metal|solution potential difference, the surface potential of the electrolyte, and the surface potential of the bare metal, respectively. Second, in eq 14, the effect of the surrounding dielectric medium outside the ligand monolayer is also ignored. Indeed, the electron addition to or removal from an MPC core is accompanied by the formation of an ionic space charge layer beyond the ligand monolayer. This space charge layer has recently been found to influences electron transfer behavior of MPCs under certain conditions. ${ }^{35-37}$ In conclusion, eq 13 provides a more rigorous definition of the standard redox potential for an MPC.

Figure 4a shows a DPV response of as-prepared MPCs in 1,2-dichloroethane electrolyte solution. The DPV trace exhibits a series of well-resolved and evenly spaced current peaks. Figure $4 \mathrm{~b}$ displays the absolute standard redox potential $\left(\left[E_{Z / Z-1}^{0}\right]_{\mathrm{abs}}\right)$ at DPV peaks as a function of the MPC charge state change. The linear relation is consistent with the prediction of eq 13 . The fitting yields a slope of $0.234 \mathrm{~V}$, which is independent of the valence state of MPCs. From the slope of the straight line, the dielectric constant of the coating layer can be estimated to be 6.2 , taking $r=0.8 \mathrm{~nm}$ and $d=0.8 \mathrm{~nm}$. This value is significantly larger than that of 3.0 previously used. ${ }^{8}$ This value may indicate that electrolyte ions and bulk solvent $\left(\epsilon_{\mathrm{s}}=10\right.$ for DCE) can penetrate into the protecting layer to some extent. ${ }^{19}$ Considering 53 hexanethiolate ligands on a surface of a 0.8 $\mathrm{nm}$ metallic ball, there exist large voids between the ligands especially at the outer boundary of the coating monolayer. Taylor dispersion measurements on the hydrodynamic radii of
MPCs have suggested the penetration of solvent into the alkanethiolate monolayer. ${ }^{19}$ More recently, the specific solvation and ionic penetration of the hexanethiolate monolayer has been inferred by investigation of the solvent and supporting electrolyte effects on the electrochemical behavior of MPC. ${ }^{37}$

Equation 13 gives the electron transfer energetics of MPCs on an absolute potential scale. However, in solution-phase electrochemical systems, the electrode potentials are normally expressed with respect to a reference electrode in the same solvent. Hence, it is necessary to correlate these two scales. In Figure $4 b$, the potential scale is referred to the absolute standard redox potential scale, which is determined experimentally by the addition of ferrocene to the solution at the end of the measurements and correlating its half-wave potential value to the absolute standard redox potential of ferrocene taken equal to $5.01 \mathrm{~V}$. This value was calculated from eqs 5 and 9 by taking $r_{\mathrm{Fc}}=r_{\mathrm{Fc}^{+}}=0.38 \mathrm{~nm}^{38}$ and $E_{\mathrm{I}, \mathrm{Fc}}^{0}=6.71 \mathrm{eV} .{ }^{39}$ It should be also noted that this absolute standard redox potential value is comparable to the value of $5.08 \mathrm{~V}$ obtained from the formal redox potential of ferrocene in DCE $\left(\left[E_{\mathrm{Fc}+/ \mathrm{Fc}}^{0^{\prime}, \mathrm{DCE}}\right]_{\mathrm{SHE}}=0.64 \mathrm{~V}\right)^{40}$ and by taking $\left[E_{\mathrm{H}^{+} / 1 / 2 \mathrm{H}_{2}}^{0}\right]_{\text {abs }}=4.44 \mathrm{~V}$. Therefore, the zero value in the potential scale in Figure 4a corresponds to an absolute potential of about $5.01 \mathrm{~V}$. According to eq 13, the intercept of the straight line for $z=0$ should be equal to $5.20 \mathrm{~V}$. Then, the peak potential value closest to $5.20 \mathrm{~V}$ on the absolute potential scale can be assigned to redox couple of $\mathrm{MPC}^{0} / \mathrm{MPC}^{-1}$. The corresponding assignment of the valence states of MPCs at the DPV peak potential is illustrated in Figure 4b.

On the basis of the above approach, the potential at the minimum of the DPV trace shown in Figure 4a corresponds to a mixture of MPCs with -2 and -3 core charges. The estimated values for $z$ are rather different from those previously determined on the basis of impedance measurements of an MPC monolayer attached to an Au electrode, in which the charged state of MPCs at the minimum of the DPV curve was assigned to be 0 , and the neighboring two peaks were assigned to the first oxidation $(z=+1 / 0)$ and reduction $(z=0 /-1)$, respectively. ${ }^{9}$

\section{Conclusions}

The redox properties of monolayer-protected gold nanoclusters (MPCs) were considered from both the theoretical and experimental viewpoints. An equation defining the "absolute standard redox potential" $\left(\left[E_{Z / Z-1}^{0}\right]_{\mathrm{abs}}\right)$ of MPCs was first derived from electrostatic considerations. The linear dependence of the standard redox potential on the valence state of MPCs (z) was verified by differential pulse voltammetry of MPCs in electrolyte solutions. The standard redox potential was related to the absolute scale by adding ferrocene to the system as the internal reference, which allows the estimation of the average charge state of MPCs at a given potential.

Acknowledgment. The work was supported by the grants from the Fonds National Suisse de la Recherche Scientifique and EPFL. We are grateful to Nicolas Eugster for reading the manuscript and Jean-Pierre Abid for HRTEM measurements. The technical assistance by Valérie Devaud is also acknowledged.

\section{References and Notes}

(1) Brust, M.; Walker, M.; Bethell, D.; Schiffrin, D. J.; Whyman, R. J. Chem. Soc., Chem. Commun. 1994, 801-802.

(2) Brust, M.; Fink, J.; Bethell, D.; Schiffrin, D. J.; Kiely, C. J. Chem. Soc., Chem. Commun. 1995, 1655-1656. 
(3) Ingram, R. S.; Hostetler, M. J.; Murray, R. W.; Schaaff, T. G.; Khoury, J.; Whetten, R. L.; Bigioni, T. P.; Guthrie, D. K.; First, P. N. J. Am. Chem. Soc. 1997, 119, 9279-9280.

(4) Chen, S.; Ingrma, R. S.; Hostetler, M. J.; Pietron, J. J.; Murray, R. W.; Schaaff, T. G.; Khoury, J. T.; Alvarez, M. M.; Whetten, R. L. Science 1998, 280, 2098-2101.

(5) Templeton, A. C.; Wuelfing, W. P.; Murray, R. W. Acc. Chem. Res. 2000, 33, 27-36.

(6) Chen, S. J. Electroanal. Chem. 2004, 574, 153-165.

(7) Pietron, J. J.; Hicks, J. F.; Murray, R. W. J. Am. Chem. Soc. 1999 $121,5565-5570$

(8) Chen, S.; Murray, R. W.; Feldberg, S. W. J. Phys. Chem. B 1998, 102, 9898-9907.

(9) Chen, S.; Murray, R. W. J. Phys. Chem. B 1999, 103, 9996-10000.

(10) Chen, S. J. Am. Chem. Soc. 2000, 122, 7420-7421.

(11) Chen, S. J. Phys. Chem. B 2000, 104, 663-667.

(12) Chen, S. Langmuir 2001, 17, 2878-2884.

(13) Chen, S. Langmuir 2001, 17, 6664-6668.

(14) Hicks, J. F.; Templeton, A. C.; Chen, S.; Sheran, K. M.; Jasti, R.; Murray, R. W.; Debord, J.; Schaaff, T. G.; Whetten, R. L. Anal. Chem. 1999, 71, 3703-3711.

(15) Hicks, J. F.; Zamborini, F. P.; Osisek, A. J.; Murray, R. W. J. Am. Chem. Soc. 2001, 123, 7048-7053.

(16) Hicks, J. F.; Miles, D. T.; Murray, R. W. J. Am. Chem. Soc. 2002, $124,13322-13328$

(17) Hicks, J. F.; Zamborini, F. P.; Murray, R. W. J. Phys. Chem. B 2002, 106, 7751-7757.

(18) Hicks, J. F.; Young, S.-S.; Murray, R. W. Langmuir 2002, 18 , $2288-2294$

(19) Wuelfing, W. P.; Templeton, A. C.; Hicks, J. F.; Murray, R. W. Anal. Chem. 1999, 71, 4069-4074.

(20) Wuelfing, W. P.; Green, S. J.; Pietron, J. J.; Cliffel, D. E.; Murray,

R. W. J. Am. Chem. Soc. 2000, 122, 11465-11472.

(21) Wuelfing, W. P.; Murray, R. W. J. Phys. Chem. B 2002, 106, 31393145 .

(22) Dubois, D.; Kadish, K. M.; Flanagan, S.; Haufler, R. E.; Chibante, L. P. F.; Wilson, L. J. J. Am. Chem. Soc. 1991, 113, 4364-4366.
(23) Dubois, D.; Moninot, G.; Kutner, W.; Jones, M. T.; Kadish, K. M. J. Phys. Chem. 1992, 96, 7137-7145.

(24) Haufler, R. E.; Conceicao, J.; Chibante, L. P. F.; Chai, Y.; Byrne, N. E.; Flanagan, S.; Haley, M. M.; O’Brien, S. C.; Pan, C.; Xiao, Z.; Billups, W. E.; Ciufolini, M. A.; Hauge, R. H.; Margrave, J. K.; Wilson, L. J.; Curl, R. F.; Smalley, R. E. J. Phys. Chem. 1990, 94, 8634-8636.

(25) Koh, W.; Dubois, D.; Kutner, W.; Jones, M. T.; Kadish, K. M. J. Phys. Chem. 1992, 96, 4163-4165.

(26) Jehoulet, C.; Bard, A. J.; Wudl, F. J. Am. Chem. Soc. 1991, 113 , $5456-5457$.

(27) Roth, J. D.; Lewis, G. J.; Jiang, X.; Dahl, L. F.; Weaver, M. J. J. Phys. Chem. 1992, 96, 7219-7225.

(28) Roth, J. D.; Lewis, G. J.; Safford, L. K.; Jiang, X.; Dahl, L. F.; Weaver, M. J. J. Am. Chem. Soc. 1992, 114, 6159-6169.

(29) Lewis, G. J.; Roth, J. D.; Montag, R. A.; Safford, L. K.; Gao, X.; Chang, S. C.; Dahl, L. F.; Weaver, M. J. J. Am. Chem. Soc. 1990, 112, $2831-2832$.

(30) Weaver, M. J.; Gao, X. J. Phys. Chem. 1993, 97, 332-338.

(31) Su, B.; Abid, J.-P.; Fermin, D. J.; Girault, H. H.; Hoffmannova, H.; Krtil, P.; Samec, Z. J. Am. Chem. Soc. 2004, 126, 915-919.

(32) Girault, H. H. Analytical and Physical Electrochemistry; EPFL Press: Lausanne, 2004 (3).

(34) Evans, S. D.; Ulman, A. Chem. Phys. Lett. 1990, 170, 462-466.

(35) Miles, D. T.; Murray, R. W. Anal. Chem. 2003, 75, 1251-1257.

(36) Quinn, B. M.; Liljeroth, P.; Ruiz, V.; Laaksonen, T.; Kontturi, K. J. Am. Chem. Soc. 2003, 125, 6644-6645.

(37) Guo, R.; Georganopoulou, D.; Feldberg, S. W.; Donkers, R.; Murray, R. W. Anal. Chem. In press.

(38) Stranks, D. R. Discuss. Faraday Soc. 1960, No. 29, 73-79.

(39) Linstrom, P. J.; Mallard, W. G. The NIST WebBook, 1998. 227. 